\title{
Fisiología del esfínter velofaríngeo durante la deglución. Revisión sistemática de literatura
}

\section{Physiology of the velopharyngeal sphincter during swallowing. Systematic review of the literature}

\author{
Felipe Inostroza-Allende ${ }^{1,2}$, Mirta Palomares-Aguilera ${ }^{1,3}$, Felipe Garrido Figueroa ${ }^{1}$, \\ Carlos Giugliano-Villarroel ${ }^{1,3,4}$
}

'Fundación Dr. Alfredo Gantz Mann. Santiago, Chile.

${ }^{2}$ Departamento de

Fonoaudiología, Facultad de

Medicina, Universidad de

Chile. Santiago, Chile.

${ }^{3}$ Smile Train - South American Medical Advisory Council (SAMAC). Santiago, Chile. `Unidad de Cirugía Plástica, Departamento de Cirugía, Clínica Alemana. Santiago, Chile.

Los autores declaran no tener conflictos de interés.

Recibido el 10 de noviembre de 2020. Aceptado el 16 de diciembre de 2020

Correspondencia: Felipe Inostroza-Allende Av. Independencia 1027, Independencia Santiago, Chile. Email: f.inostrozarp@gmail.

com

\section{Resumen}

El objetivo de este artículo es describir el funcionamiento del esfínter velofaríngeo (EVF) durante la deglución, mediante una revisión de literatura. En febrero de 2020, las bases de datos electrónicas Medline, LILACS, SciELO e IBECS, fueron consultadas retrospectivamente, usando las palabras claves en inglés: "velopharyngeal sphincter" o "pharyngeal muscles". Fueron seleccionados artículos originales que describen la fisiología del EVF en la deglución de adultos sanos. Para este estudio fue creado un protocolo que contempla lo siguiente: autor, año, país, número y características de los participantes, actividades evaluadas, metodologías e instrumentos utilizados y principales resultados. Fueron encontrados 4.124 artículos. 3.863 fueron excluidos luego de la lectura de los títulos, 239 luego de lectura de los resúmenes y 8 luego de la revisión de los textos completos. Finalmente, 14 artículos fueron analizados en esta revisión. Se discuten eventos espaciales y temporales del EVF, la actividad electromiográfica de la musculatura del EVF y la presión velofaríngea durante la deglución en adultos sanos. Se concluye que el esfínter velofaríngeo cumple un rol importante en la fase faríngea de la deglución, que debe ser profundizado en futuras investigaciones.

Palabras clave: Esfínter velofaríngeo, músculos faríngeos, deglución, fisiología.

\begin{abstract}
The aim of this article is to describe the functioning of the velopharyngeal sphincter (VPS) during swallowing, through a literature review. In February 2020, the electronic databases Medline, LILACS, SciELO and IBECS were retrospectively consulted, using the key words in English: "velopharyngeal sphincter" or "pharyngeal muscles". Original articles were selected that describe the physiology of VPS in healthy adult swallowing. For this study it was created a protocol that includes the following items: author, year, country, number and characteristics of the participants, evaluated activities, methodologies and instruments used, and main results. 4124 articles were found. 3,863 were excluded after reading the titles, 239 after reading the abstracts and 8 after reviewing the full texts. Finally, 14 articles were analyzed in this review. Spatial and temporal events of the VPS, the electromyographic activity of the VPS musculature, and velopharyngeal pressure during swallowing in healthy adults are discussed. We conclude that the velopharyngeal sphincter plays an important role in the pharyngeal phase of swallowing, which should be studied in depth in future research. Keywords: Velopharyngeal sphincter, pharyngeal muscles, deglutition, physiology.
\end{abstract}

\section{Introducción}

El esfínter velofaríngeo (EVF) corresponde a un grupo de músculos unidos al paladar blando (o velo del paladar) y a la faringe, que constituyen una válvula muscular situada entre las cavidades oral y nasal. Su función es crear un sello hermético entre el velo y las paredes faríngeas para separar las cavidades oral y nasal. Un esfínter velofaríngeo competente es 
esencial para una respiración, habla, succión y deglución adecuadas ${ }^{1,2}$.

El EVF está delimitado anteriormente por el paladar blando, o velo del paladar; por las paredes laterales de la faringe $y$, posteriormente, por la pared posterior de la faringe. Este esfínter está compuesto por seis tipos de músculos: el elevador del velo del paladar, el tensor del velo del paladar, el músculo de la úvula, el palatogloso, el palatofaríngeo y el constrictor superior de la faringe $e^{3}$.

En la respiración nasal, la superficie oral del velo del paladar está descendida y descansa sobre la parte posterior de la base de la lengua ${ }^{1}$. El estudio del EVF en la respiración, se ha orientado principalmente al síndrome de apnea obstructiva del sueño (SAOS). Al respecto, diversos autores han descrito cambios del EVF en sujetos con SAOS, mediante análisis histológico de los músculos ${ }^{4}$, inmunohistoquímicos e histomorfométricos de su inervación ${ }^{5}$.

Durante el habla y la deglución, el velo del paladar y las paredes de la faringe se aproximan para tener un cierre completo. En el habla, mediante la visualización directa del EVF con nasofaringoscopía y videofluoroscopía durante la producción de sonidos orales, se pueden observar múltiples patrones de cierres (coronal, sagital, circular y circular con rodete de Passavant), en donde cada pared del EVF tiene diferentes grados de participación ${ }^{6}$. El estudio funcional del mecanismo velofaríngeo durante el habla ha sido de gran interés debido a sus implicancias clínicas en la insuficiencia velofaríngea congénita o adquirida. En la literatura destacan evaluaciones aerodinámicas ${ }^{7} \mathrm{y}$ estudios electromiográficos durante el habla, principalmente del músculo elevador del velo del paladar ${ }^{8}$. Menos utilizada, la resonancia magnética tridimensional se ha empleado en la descripción de la fisiología del EVF en el habla9 .

La función del esfínter velofaríngeo durante la alimentación aún no está totalmente esclarecida. Los movimientos rítmicos del paladar blando durante la masticación están vinculados temporalmente al movimiento de la mandíbula, mientras que durante la fase faríngea de la deglución ocurre un cierre completo del EVF, lo cual evita la regurgitación nasal de los alimentos ${ }^{10}$. Sin embargo, se desconoce qué otras actividades desarrolla el EVF durante la deglución. Debido a esto, nace el interés de describir la fisiología del esfínter velofaríngeo durante la deglución, mediante una revisión sistemática de literatura. Esta revisión se desarrolló de acuerdo con la lista de verificación de la declaración PRISMA (elementos de informe preferidos para revisiones sistemáticas y metaanálisis $)^{11}$.

\section{Estrategia de búsqueda}

Este estudio trata de una revisión con la finalidad de reunir y sintetizar resultados de investigaciones sobre el tema, de modo sistemático y ordenado. La elaboración de ésta se basó en la pregunta: ¿qué sabemos sobre la fisiología del esfínter velofaríngeo durante la deglución?

En febrero de 2020, se realizó una búsqueda electrónica sin límite de tiempo en las siguientes bases de datos: Medical Literature Analysis and Retrieval System Online (Medline), Literatura Latino-Americana e do Caribe em Ciências da Saúde (LILACS), Scientific Electronic Library Online (SciELO) e Índice Bibliográfico Español en Ciencias de la Salud (IBECS). Para la búsqueda de los artículos se utilizaron términos claves en inglés, disponibles en Medical Subject Headings (MeSH), una plataforma con vocabulario terminológico controlado para publicaciones de artículos y libros de ciencia. Se utilizaron independientemente los conceptos MeSH: "velopharyngeal sphincter" OR "pharyngeal muscles".

\section{Criterios de selección}

Los criterios de elegibilidad definidos para la selección de los artículos fueron: serie de casos, estudio de cohorte, estudio de casos y controles, ensayo clínico sin asignación aleatoria y aleatorizados, que reportan y describen la fisiología del esfínter velofaríngeo durante la deglución mediante electromiografía y manometría de alta resolución, entre otros, en adultos sanos mayores de 18 años. Se excluyeron trabajos en niños y adolescentes, estudios en sujetos con disfagia o fisura de paladar, revisiones de literatura, presentaciones en congresos, resúmenes, tesis, capítulos de libro y artículos de estudio de caso único. 


\section{Extracción y análisis de los datos}

Para la selección de los estudios, dos de los investigadores realizaron de manera independiente y paralela, la búsqueda, lectura y selección por los títulos y resúmenes de las publicaciones localizadas en las bases de datos electrónicas, en los casos de discrepancia fue consultado un tercer investigador. En seguida, se procedió a la lectura de los artículos seleccionados, que cumplieron los criterios de selección y que permiten responder a la pregunta de esta investigación. En esta etapa, cada artículo fue evaluado por dos de los autores y, en caso de duda, hubo consenso entre todos los autores. Por último, la información descriptiva de los artículos seleccionados fue sistemáticamente extraída y analizada, a través de un protocolo diseñado antes de la revisión, el cual contempla los siguientes puntos: autor, año, país, número, edad y sexo biológico de los participantes, equipamientos, procedimientos de evaluación, análisis estadístico y principales resultados.

\section{Revisión de la literatura}

Utilizando el algoritmo de búsqueda fueron encontrados 4.124 artículos. Se excluyeron 3.863 por el título debido a que el contenido no se refería al funcionamiento del esfínter velofaríngeo durante la deglución, 239 luego de lectura de los resúmenes porque los parti-

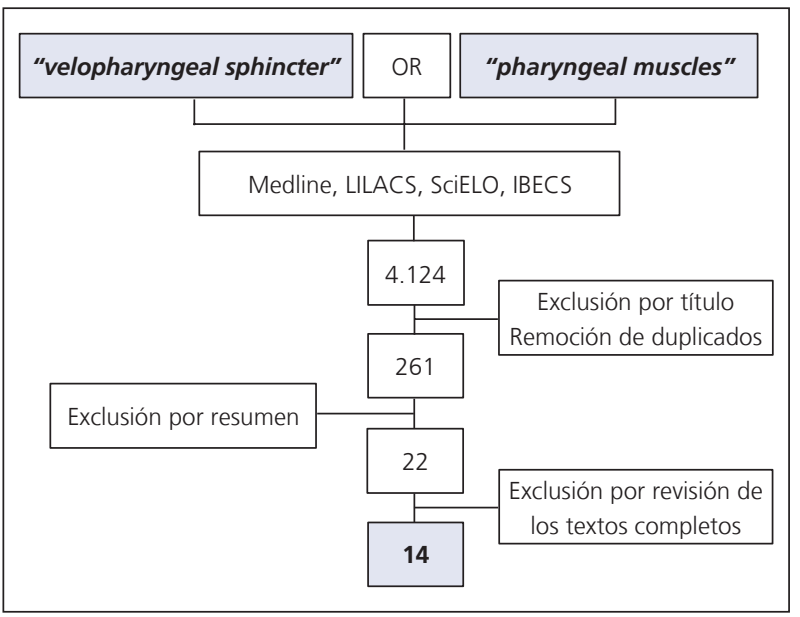

Figura 1. Proceso de selección de estudios. cipantes y las metodologías de evaluación no cumplieron con los criterios de selección y 8 posterior a la revisión de los textos completos, ya que los diseños y resultados no permitían responder a la pregunta de investigación. Finalmente, 14 artículos fueron analizados en esta revisión. La Figura 1 resume el proceso de selección de los estudios. La Tabla 1 presenta el primer autor, año de publicación, país, número, sexo biológico y edad de los participantes. En un análisis descriptivo de los artículos, se aprecia que la mayoría de los estudios incluidos corresponden al siglo XXI ${ }^{14-25}$.

Las investigaciones fueron en su mayoría realizadas en Japón ${ }^{15,16,20-22,25}$ y Estados Uni$\operatorname{dos}^{12,13,18,19,23,24}$. El número de muestra mínimo fue de 2 individuos ${ }^{12,23}$ y el máximo de $67^{25}$. Los instrumentos de evaluación consistieron en rayos $\mathrm{X}$, videofluoroscopía, electromiografía intramuscular, electromiografía de superficie, tomografía computacional tridimensional y manometría de alta resolución. A continuación, los resultados de la búsqueda son clasificados en estudios sobre: el movimiento del EVF durante la deglución, la actividad electromiográfica de los músculos del EVF durante la deglución y la presión velofaríngea durante la deglución.

\section{Movimiento del esfínter velofaríngeo durante la deglución}

En relación con el análisis temporal y espacial del movimiento de las estructuras velofaríngeas durante la deglución, Hamlet y Momiyama (1992) evaluaron a 2 sujetos adultos sanos de 25 y 49 años. Los investigadores registraron el movimiento velar mediante el seguimiento radiográfico de un sedimento de oro de 2,5 milímetros ( $\mathrm{mm}$ ) suturado a la superficie lingual del paladar blando, y midieron la actividad electromiográfica (EMG) de los músculos elevador del velo del paladar (EVP) y tensor del velo del paladar (TVP), durante tareas de: deglución en seco y de 10 milímetros (ml) de agua; y habla (repetición de una frase) de forma pseudoaleatoria. Entre los principales resultados destaca que las trayectorias del movimiento velar en el habla y la deglución son diferentes, tanto en extensión como en forma. En el habla, los movimientos fueron casi lineales, 


\begin{tabular}{|c|c|c|c|c|c|}
\hline Primer autor & Año & País & $\mathrm{N}$ & Sexo & Edad \\
\hline Hamlet $^{12}$ & 1992 & USA & 2 & NE & 25 y 49 a \\
\hline Kahrilas $^{13}$ & 1996 & USA & 8 & $8 \mathrm{M}$ & $22-37 a$ \\
\hline Picciotti14 & 2005 & Italia & 10 & $5 M-5 F$ & $27-37 a$ \\
\hline Tachimura ${ }^{15}$ & 2005 & Japón & 7 & $3 M-4 F$ & $26,9 \pm 2,9 a$ \\
\hline Tachimura $^{16}$ & 2006 & Japón & 8 & $4 M-4 F$ & $26,1 \pm 3,13 a$ \\
\hline Takasaki17 & 2008 & Japón & 33 & $19 M-14 F$ & $22-29 a$ \\
\hline Hoffman ${ }^{18}$ & 2010 & USA & 12 & $5 M-7 F$ & $20,9 \pm 1,8 a$ \\
\hline Hoffman ${ }^{19}$ & 2012 & USA & 14 & $7 M-7 F$ & $21,2 \pm 2,0 a$ \\
\hline Okuno 20 & 2013 & Japón & 9 & $5 M-4 F$ & $25,3 \pm 2,2 a$ \\
\hline Matsubara21 & 2014 & Japón & 30 & $15 M-15 F$ & $25,3 \pm 3,6 a$ \\
\hline Matsubara22 & 2015 & Japón & 26 & $10 M-16 F$ & $21-35 a$ \\
\hline Hutcheson ${ }^{23}$ & 2017 & USA & 2 & $2 \mathrm{~F}$ & 22 y 24 a \\
\hline Rosen ${ }^{24}$ & 2017 & USA & 10 & $3 M-7 F$ & $20-51 a$ \\
\hline Pongpipatpaiboon 25 & 2018 & Japón & 67 & $\mathrm{NE}$ & $20-74$ \\
\hline
\end{tabular}

con cambios predominantemente verticales; mientras que para la deglución se identificaron dos tipos de patrón en las trayectorias velares. La velocidad máxima promedio asociada con el movimiento de deglución fue mayor que la encontrada para la elevación velar en el habla. No hubo diferencias estadísticamente significativas en la duración de la contracción de ninguno de los músculos durante la deglución en seco y de líquidos ${ }^{12}$.

Kahrilas y cols. (1996) evaluaron ocho sujetos masculinos sanos (edad entre 22 a 37 años). Los investigadores utilizaron imágenes fluorográficas, en los planos anteroposterior y lateral para realizar un análisis temporal y espacial del movimiento de las estructuras velofaríngeas durante la deglución de una mezcla de bario líquido y agua en dos volúmenes: 1 y $20 \mathrm{ml}$. Para realizar el análisis temporal se utilizó un videotímero (modelo VC 436; Thalner Electronics Laboratories, Ann Arbor, MI), que codificó las cintas de video en una señal de temporización numérica en centésimas de segundo. El análisis espacial se llevó a cabo mediante el seguimiento de coordenadas $(\mathrm{x}, \mathrm{y})$ de las estructuras seleccionadas en los planos anteroposterior y lateral. En los resul- tados se observa un aumento en el tiempo de cierre del EVF durante la deglución de $20 \mathrm{ml}$ $(1,13 \pm 0,04$ segundos), frente a la deglución de $1 \mathrm{ml}(0,93 \pm 0,03$ segundos). Destaca que la reconfiguración faríngea es 0,2 segundos más larga en la deglución de $20 \mathrm{ml}$ en comparación con la deglución de $1 \mathrm{ml}$, sin embargo, la sincronía entre las válvulas con respecto al inicio $\mathrm{y}$ al final de la reconfiguración no cambia ${ }^{13}$.

Por último, el tercer estudio incluido fue el de trabajo de Pongpipatpaiboon y cols. (2018). Los investigadores evaluaron la deglución faríngea de un líquido espeso tipo miel gruesa $(1700 \mathrm{mPa})$ de $10 \mathrm{ml}$ utilizando una tomografía computarizada tridimensional mediante scanner 320-ADCT. Los participantes fueron adultos divididos en tres grupos etarios: adultos jóvenes (20 a 39 años), adultos de mediana edad (40 a 59 años) y adultos mayores (60 a 74 años). En los resultados observaron que, en adultos jóvenes, la apertura velofaríngea ocurrió antes de la apertura completa del esfínter esofágico superior. En los adultos mayores el cierre velofaríngeo tuvo una duración prolongada, que los autores asocian a un mecanismo compensatorio de la debilidad relacionada con la edad ${ }^{25}$. 
Actividad electromiográfica de la musculatura velofaríngea durante la deglución

Las señales electromiográficas de diferentes músculos del EVF durante la deglución fueron descritas en 4 estudios. Picciotti y cols. (2005) analizaron la actividad electromiográfica bilateral del músculo elevador del velo del paladar durante la deglución en 10 sujetos sanos, de entre 27 a 37 años. En cada sujeto se registraron y analizaron al menos cuatro activaciones EMG inducidas por degluciones consecutivas. La captación de la señal se obtuvo utilizando un electrodo nasofaríngeo metálico casero (largo de $16 \mathrm{~cm}$ y diámetro de $1,5 \mathrm{~mm}$ ) acoplado a un polígrafo digital (Micromed System 98). Los resultados obtenidos muestran que la deglución siempre induce una activación EMG repetitiva y polifásica, que dura de 2 a 4,5 segundos, con una amplitud máxima de 250 microvoltios $(\mu \mathrm{V})$. El análisis frecuencial realizado por fast Fourier transformed, mostró un pico de frecuencia de $180 \mathrm{~Hz}$ en la actividad basal y un pico de frecuencia de $240 \mathrm{~Hz}$ durante la deglución ${ }^{14}$.

Ese mismo año, Tachimura y cols. (2005), evaluaron señales electromiográficas de los músculos palatogloso (PG) y elevador del velo del paladar (EVP) en siete adultos sanos (edad $=26,9 \pm 2,9$ años), durante la deglución de 5 diferentes volúmenes de agua: 12,5\%, $25 \%, 50 \%, 100 \%$ y $150 \%$ (o $200 \%$ ) del volumen óptimo para deglutir, determinado para cada sujeto. La actividad electromiográfica se monitoreo utilizando electrodos bipolares. Para ello, se insertaron dos agujas en el músculo PG izquierdo en el punto medio vertical del pilar anterior, y dos agujas en el músculo EVP izquierdo. Se realizó un análisis temporal de la forma de onda EMG del músculo PG en relación con la actividad muscular del EVP, y se evaluó la amplitud de los picos de la curva EMG del músculo PG. A nivel temporal, las formas de ondas del músculo PG para una sola deglución tenían uno o dos picos para todos los sujetos. En el caso de un pico único, este usualmente apareció simultáneamente con el músculo EVP. En el caso de dos picos, el segundo apareció de una manera similar a la del pico único, mientras que el primer pico aparece antes de que el músculo EVP se acti- vara. La actividad del músculo PG en el primer pico fue variable en todos los sujetos. Mientras que la actividad muscular del PG observada en el segundo pico fue significativamente mayor que en el primero, independientemente del volumen deglutido ${ }^{15}$.

$\mathrm{Al}$ año siguiente, Tachimura y cols. (2006), presentaron los resultados de ocho adultos sanos (edad $=26,1 \pm 3,13$ años). En esta oportunidad, nuevamente reportaron las señales electromiográficas de los músculos PG y EVP durante la deglución de los mismos volúmenes de agua del estudio anterior. En el análisis, las amplitudes del EVP y PG se expresaron como porcentajes relativos al valor máximo de la actividad EMG observada a lo largo del experimento. La actividad del PG también se controló para evaluar si había coordinación entre la actividad muscular del EVP y el PG. Los resultados presentados se refieren principalmente a la actividad EMG del músculo EVP. Entre los hallazgos destaca que los cambios en la actividad muscular del EVP se correlacionan positivamente y de forma significativa con el aumento del volumen ingerido. Además, algunos adultos mostraron correlaciones significativas entre las actividades musculares del PG y el EVP ${ }^{16}$.

Más recientemente, Okuno, Tachimura y Sakai (2013) evaluaron señales electromiográficas del músculo elevador del velo del paladar (EVP) en nueve adultos sanos (edad $=25,3 \pm 2,2$ años), durante la deglución de nueve alimentos variados en volumen y viscosidad. Se utilizaron tres volúmenes de té verde: $1 / 4,1 / 2$ y 1 del volumen óptimo para deglutir, determinado para cada sujeto. La viscosidad fue ajustada a 0,0, 2,0 y 4,6 Pa/s mediante la mezcla con espesante. Cada sujeto realizó al azar 4 a 5 ensayos para cada alimento de prueba. Al igual que en sus estudios anteriores, los autores monitorearon la actividad electromiográfica del músculo EVP utilizando electrodos bipolares. Se determinó la media del nivel de actividad muscular durante la ingesta de cada alimento de prueba. Sus resultados muestran que la actividad del músculo EVP aumenta con el volumen deglutido para todos los sujetos y disminuye inversamente con la viscosidad para seis de los sujetos, pero no cambia con el incremento de la viscosidad para tres de los sujetos ${ }^{20}$. 


\section{Presión velofaríngea durante la deglución}

La introducción del manómetro de alta resolución (MAR) durante la deglución, ha permitido el estudio de la presión a nivel de la velofaringe, la base de lengua o mesofaringe, la hipofaringe y del esfínter esofágico superior (EES). Takasaki y cols. (2008) estudiaron los valores de presiones deglutorias desde la velofaringe hasta la parte superior del esófago en 33 japoneses (19 hombres y 14 mujeres, con rango de edad entre 22 a 29 años) sin historia de alteraciones deglutorias y/o gástricas. En su estudio utilizaron un MAR (Sierra Scientific Instruments, Los Ángeles, CA) durante degluciones en seco y de $5 \mathrm{ml}$ de agua fría $\left(0^{\circ} \mathrm{C}\right)$. Los resultados mostraron que al deglutir $5 \mathrm{ml}$ de agua fría la presión velofaríngea fue mayor que al tragar en seco. En tanto, la presión máxima ( $\mathrm{mm} \mathrm{Hg}$ ) de la velofaringe no se diferenció entre hombres y mujeres ${ }^{17}$.

En otro estudio, Hoffman y cols. (2010) evaluaron a 5 hombres y 7 mujeres (edad $=20,9$ $\pm 1,8$ años) sin alteraciones de la deglución. Se utilizó un MAR (ManoScan360 High Resolution Manometry System, Sierra Scientific Instruments, Los Ángeles, CA) durante la deglución de cuatro volúmenes de bolos: saliva, 5,10 y $20 \mathrm{ml}$ de agua. Los resultados evidenciaron que la duración del tránsito faríngeo y la presión máxima de la velofaringe aumentan de manera directa con el volumen del bolo. Por su parte, el sexo no tuvo ningún efecto sobre las presiones ${ }^{18}$.

En la misma línea de investigación, Hoffman y cols. (2012) evaluaron a 7 hombres y 7 mujeres sanos (edad $=21,2 \pm 2,0$ años). Los investigadores utilizaron el mismo equipo de MAR para determinar las presiones de la velofaringe, la base de lengua y el EES, durante la deglución de cinco bolos de agua de $5 \mathrm{ml}$, en cada una de las siguientes tareas: deglución normal, deglución con esfuerzo y la maniobra de Mendelsohn. En los resultados observaron que la deglución con esfuerzo aumentó la presión máxima de la velofaringe $(\mathrm{p}=0,020)$. La maniobra de Mendelsohn disminuyó la tasa de aumento de la presión velofaríngea $(\mathrm{p}=0,027)$ y aumentó significativamente $(\mathrm{p}<0,001)$ la duración de la presión velofaríngea por encima de la línea de base ${ }^{19}$.
Con el mismo sistema de MAR (ManoScan 360; Given/Sierra Scientific Instruments, Mountain View, CA), Matsubara y cols. (2014) obtuvieron valores de presión deglutoria máxima de la velofaringe, mesofaringe, hipofaringe, EES y esófago cervical, en 30 adultos jóvenes sanos (edad $=25,3 \pm 3,6$ años), durante la deglución de saliva, agua fría y agua caliente en cantidades de 2, 5 y $10 \mathrm{ml}$. Los resultados indican que estas variantes no alteran los valores de presión en la velofaringe. Por otra parte, la presión velofaríngea fue mayor en hombres versus mujeres, para la deglución de saliva $(\mathrm{p}=0,0029)$; de agua caliente: 2 $\mathrm{ml}(\mathrm{p}=0,0148), 5 \mathrm{ml}(\mathrm{p}=0,0352)$ y $10 \mathrm{ml}$ $(\mathrm{p}=0,1175)$; $y$ de agua fría: $5 \mathrm{ml}(\mathrm{p}=0,0329)$ y $10 \mathrm{ml}(\mathrm{p}=0,0259)^{21}$.

A continuación, Matsubara y cols. (2016) determinaron los valores de presión máxima en la región velofaríngea, mesofaringe, hipofaringe y el EES, durante la deglución de $5 \mathrm{ml}$ de agua fría, en una posición neutra como control y en 3 diferentes maniobras de chin-down: 1) flexión de la cabeza; 2) flexión del cuello; y 3) flexión combinada de cabeza y cuello. En el estudio participaron 26 adultos jóvenes sanos (edad entre 21 y 35 años) y utilizaron el mismo equipo de MAR de su estudio anterior. $\mathrm{Al}$ respecto, la presión velofaríngea no se diferenció entre la posición neutra y las 3 diferentes maniobras de chin-down ${ }^{22}$.

Similar al artículo anterior, Rosen y cols. (2017) realizaron un estudio sobre los efectos de cambios de posición corporal en la presión deglutoria mediante uso de MAR (ManoScan360 High-Resolution Manometry System, Medtronic, MN). Participaron 10 sujetos, 3 hombres y 7 mujeres con edad promedio de 27 años. Los sujetos ingirieron 3 bolos de agua de $5 \mathrm{ml}$ cada uno, mientras se sometían a grados de inclinación corporal: $0^{\circ}$ (vertical), $45^{\circ}, 90^{\circ}$ (supino), $110^{\circ}, 135^{\circ}$ y $180^{\circ}$ (completamente invertido). Los resultados mostraron que la presión velofaríngea aumenta significativamente $(\mathrm{p}<0,001)$ con una inclinación mayor o igual a $90^{\circ}$, mientras que la duración de la presión no se vio afectada por la posición. La presión velofaríngea aumenta durante la posición en $180^{\circ}$ para lograr el cierre nasofaríngeo y prevenir la regurgitación nasal2 ${ }^{24}$.

Finalmente, Hutcheson y cols. (2017) obtuvieron datos de manometría faríngea de 
ARTÍCULO DE REVISIÓN

Tabla 2. Movimiento del esfínter velofaríngeo durante la deglución

\begin{tabular}{|c|c|c|}
\hline Primer autor & Actividades evaluadas & Principales resultados \\
\hline Hamlet $^{12}$ & $\begin{array}{l}\text { Análisis temporal y espacial del movi- } \\
\text { miento velar y la actividad EMG de los } \\
\text { músculos EVP y TVP durante tareas de } \\
\text { deglución y habla }\end{array}$ & $\begin{array}{l}\text { Se identificaron dos tipos de patrón en las trayectorias } \\
\text { velares durante la deglución. La velocidad durante la } \\
\text { deglución fue mayor que la encontrada para la elevación } \\
\text { velar en el habla }\end{array}$ \\
\hline Kahrilas $^{13}$ & $\begin{array}{l}\text { Análisis fluorográfico del movimiento } \\
\text { de las estructuras velofaríngeas durante } \\
\text { tareas de deglución con } 1 \text { y } 20 \mathrm{ml}\end{array}$ & $\begin{array}{l}\text { El tiempo de cierre VF fue mayor durante la deglución de } \\
20 \mathrm{ml} \text {. En tanto, existió una sincronía entre las válvulas } \\
\text { con respecto al inicio y final de la reconfiguración }\end{array}$ \\
\hline Pongpipatpaiboon ${ }^{25}$ & $\begin{array}{l}\text { Análisis por TC 3D de la función faríngea } \\
\text { durante la deglución de líquido espeso } \\
\text { tipo miel gruesa en } 3 \text { rangos etarios }\end{array}$ & $\begin{array}{l}\text { Los tiempos de apertura y cierre del EVF y EES variaron a } \\
\text { medida que aumentó la edad. La apertura VF ocurrió en } \\
\text { menor tiempo en adultos jóvenes y con mayor desfase } \\
\text { en los adultos mayores }\end{array}$ \\
\hline
\end{tabular}

Abreviaciones: EVP = elevador del velo del paladar; TVP = tensor del velo del paladar; EMG = electromiográfica; $\mathrm{ml}=$ mililitros; $\mathrm{EVF}=$ esfínter velofaríngeo; EES = esfínter esofágico superior; $\mathrm{TC}$ 3D = tomografía computarizada tridimensional; $\mathrm{VF}=$ velofaríngea.

alta resolución (ManoScan360 High-Resolution Manometry System, Given Imaging, Atlanta, GA) y EMG intramuscular (palatal, laríngea y faríngea) en 2 mujeres jóvenes sanas (edad $=22$ y 24 años), durante cinco tareas: deglución de 10 centímetros cúbicos (cc) de agua, prueba de presión espiratoria máxima, y entrenamiento de fuerza muscular espiratoria en tres niveles de presión (50\%, 75\% y simulado). Se registraron y analizaron veinticinco ensayos (cinco por cada tarea), con interés en la presión velofaríngea. La actividad electromiográfica en el músculo elevador del paladar (EVP), los músculos faríngeos y el músculo tiroaritenoideo fue adquirida con electrodos intramusculares bipolares de alambre. Las señales EMG se amplificaron, filtraron y digitalizaron. El dispositivo de entrenamiento respiratorio fue el EMST (EMST150, Aspire Products, Gainesville, FL). En los resultados destaca que, para todas las tareas, hubo un aumento en la actividad EMG de cada músculo, en comparación con el valor inicial. La actividad EMG del músculo EVP generalmente aumentó de una manera dependiente de la carga espiratoria. El patrón de actividad EMG del músculo EVP fue paralelo al aumento de la presión de cierre en la velofaringe, detectada en la manometría. Las tareas espiratorias de alta intensidad generaron amplitudes máximas en la señal EMG del músculo EVP, equivalentes o superiores a las generadas durante las tareas de deglución. Por el contrario, la presión velofaríngea durante tareas espiratorias simuladas se encontró en niveles inferiores a los observados durante la deglución de $10 \mathrm{cc}$. Por su parte, la actividad EMG del músculo faríngeo aumentó con la carga del esfuerzo espiratorio ${ }^{23}$. Las Tablas 2,3 y 4 resumen las actividades evaluadas y los principales resultados de los 14 estudios analizados en esta revisión.

\section{Discusión}

El conocimiento respecto al funcionamiento del esfínter velofaríngeo es fundamental para la evaluación y rehabilitación de sus alteraciones. $\mathrm{Al}$ respecto, esta revisión tuvo el objetivo de describir la fisiología del esfínter velofaríngeo durante la deglución, mediante una revisión sistemática de literatura. La preocupación por el estudio de la fisiología del EVF durante la deglución inicia en la década del $1990^{12,13}$, con una mayor intensificación a partir del año $2005^{14,15}$. Lo cual está relacionado con el desarrollo y acceso de nuevos equipos tecnológicos y sistemas computacionales.

Respecto al análisis temporal y espacial del movimiento de las estructuras velofaríngeas durante la deglución, el estudio videofluoroscópico de la deglución (videofluorographic swa- 


\section{Tabla 3. Actividad electromiográfica de la musculatura velofaríngea durante la deglución}

\begin{tabular}{|c|c|c|}
\hline Primer autor & Actividades evaluadas & Principales resultados \\
\hline Picciotti $^{14}$ & $\begin{array}{l}\text { Actividad EMG bilateral del músculo } \\
\text { EVP durante degluciones consecutivas }\end{array}$ & $\begin{array}{l}\text { La deglución indujo una activación EMG que dura de } 2 \text { a } 4,5 \mathrm{~s} \text {, } \\
\text { con una amplitud máxima de } 250 \mu \mathrm{V} \text {. El análisis frecuencial, } \\
\text { mostró una diferencia pico de } 60 \mathrm{~Hz} \text { entre la actividad basal y } \\
\text { la deglución }\end{array}$ \\
\hline Tachimura ${ }^{15}$ & $\begin{array}{l}\text { Análisis EMG de los músculos PG } \\
\text { y EVP durante la deglución de } 5 \\
\text { diferentes volúmenes de agua, del } \\
\text { volumen óptimo para deglutir }\end{array}$ & $\begin{array}{l}\text { La forma de onda del músculo PG se caracterizó por la presencia } \\
\text { de uno o dos picos durante la deglución. La amplitud del primer } \\
\text { pico fue variable, mientras que la del segundo fue mayor que el } \\
\text { primero independientemente del volumen de deglución }\end{array}$ \\
\hline Tachimura $^{16}$ & $\begin{array}{l}\text { Misma actividad que Tachimura } \\
(2006)^{15}\end{array}$ & $\begin{array}{l}\text { La actividad muscular del EVP se correlacionó positivamente con } \\
\text { el aumento del volumen ingerido. La actividad EMG del PG y EVP } \\
\text { se correlacionó en algunos adultos }\end{array}$ \\
\hline Okuno 20 & $\begin{array}{l}\text { Análisis EMG del músculo EVP } \\
\text { durante la deglución de } 9 \text { alimentos } \\
\text { variados en volumen y viscosidad }\end{array}$ & $\begin{array}{l}\text { La actividad del músculo EVP aumenta con el volumen deglutido } \\
\text { para todos los sujetos y disminuye inversamente con la } \\
\text { viscosidad para seis de los sujetos }\end{array}$ \\
\hline
\end{tabular}

Tabla 4. Presión velofaríngea durante la deglución

$\begin{array}{ll}\text { Primer autor } & \text { Actividades evaluadas } \\ \text { Takasaki }^{17} & \text { Análisis con MAR durante la deglución } \\ & \text { en seco y de } 5 \mathrm{ml} \text { de agua fría }\left(0^{\circ}\right)\end{array}$

Hoffman ${ }^{18} \quad$ Análisis con MAR durante la deglución en seco, 5, 10 y $20 \mathrm{ml}$ de agua

$\begin{array}{ll}\text { Hoffman }^{19} & \text { Análisis con MAR durante la deglución } \\ \text { de bolos de agua de } 5 \mathrm{ml} \text {, de forma } \\ \text { normal, con esfuerzo y la maniobra de } \\ \text { Mendelsohn }\end{array}$

Matsubara ${ }^{21} \quad$ Análisis con MAR durante la deglución en seco y de agua, a diferentes volúmenes y temperaturas
Matsubara22 Análisis con MAR durante la deglución de $5 \mathrm{ml}$ de agua fría, utilizando una posición neutra y tres posturas de chin down

$\begin{array}{ll}\text { Rosen }^{24} & \text { Análisis con MAR durante la deglución } \\ \text { de } 5 \mathrm{ml} \text { de agua, utilizando distintos } \\ \text { grados de inclinación }\end{array}$

Hutcheson ${ }^{23}$ Análisis con MAR y EMG intramuscular (palatal, laríngea y faringe) durante tareas de deglución y entrenamiento respiratorio

\section{Principales resultados}

La presión VF fue mayor en la deglución de agua fría. En tanto, la presión VF máxima no se diferenció entre hombres y mujeres

La duración del tránsito faríngeo y la presión VF máxima aumentó directamente con el volumen del bolo alimenticio. El sexo no tuvo efecto en las variables

La deglución con esfuerzo aumentó la presión VF máxima. La maniobra de Mendelsohn disminuyó la tasa de aumento de la presión VF y aumentó la duración de la misma

Las diferencias de volumen y temperatura del bolo no modificaron la presión VF. En tanto, ésta fue mayor en hombres que en mujeres para la deglución en seco, de agua caliente a 2 , 5 y $10 \mathrm{ml}$, y en el agua fría a 5 y $10 \mathrm{ml}$

La presión VF no se diferenció entre la posición neutra y las tres maniobras de chin down evaluadas

La presión VF aumentó en inclinaciones iguales o mayor a $90^{\circ}$. En tanto, a más de $180^{\circ}$ ésta aumentó para prevenir la regurgitación nasal. La duración no se afectó con los cambios de inclinación

En todas las tareas aumentó la actividad EMG de cada músculo. La actividad EMG del EVP y la presión VF se relacionaron directamente. Las tareas espiratorias de alta intensidad aumentaron la señal EMG del EVP y del músculo faríngeo

Abreviaciones: $M A R=$ manómetro de alta resolución; VF = velofaríngea; $\mathrm{ml}=$ mililitros; $\mathrm{EMG}=$ electromiográfica; EVP = elevador del velo del paladar. 
llowing study, VFSS) es una de las herramientas de evaluación instrumental más utilizadas para determinar la naturaleza y extensión de un trastorno de la deglución orofaríngea. El VFSS determina los detalles de la disfunción de la deglución orofaríngea y ayuda a orientar las decisiones con respecto a la terapia de la deglución según estos hallazgos. La elevación del paladar blando para el sello nasofaríngeo está incluida en los protocolos para el VFSS, por lo que su estudio en adultos sanos es fundamental para la comparación con los trastornos de la deglución orofaríngea ${ }^{26}$. En esta revisión, los estudios incluidos describen la trayectoria, velocidad y sincronía del movimiento velar, así como el efecto del volumen ingerido en el tiempo de cierre del EVF.

En relación a las señales electromiográficas del EVF durante la deglución, podemos señalar que la actividad electromiográfica también ha sido utilizada para contrastar la función muscular de adultos sanos y adultos con disfagia. En el año 2016, un estudio realizado en 70 sujetos, 50 clasificados con disfagia y 20 sanos (edad $=67,30 \pm 13,1$ años), registró y analizó señales EMG de superficie nasofaríngeas, inducidas por degluciones consecutivas. En los resultados se observó que la duración de la señal EMG aumentó en los pacientes con disfagia con una diferencia estadísticamente significativa en comparación con los pacientes sin disfagia $(p<0,001)$. La amplitud se redujo ligeramente en el grupo de disfágicos, pero no se observaron diferencias estadísticamente significativas ( $p=0,775)$. Sin embargo, la amplitud de la ráfaga mostró una correlación inversa significativa con la gravedad clínica y la extensión topográfica de las lesiones cerebrales en la tomografía computarizada ${ }^{27}$.

$\mathrm{Al}$ respecto, los estudios de la señal EMG de la musculatura velofaríngea en individuos con deglución normal entregan información importante sobre la fisiología de la deglución. Estos resultados podrían ser útiles para el manejo clínico de la disfagia orofaríngea. En tanto, el sistema de manometría de alta resolución también ha sido utilizado en sujetos con disfagia. Un estudio evaluó 12 sujetos sanos (edad $=20,9 \pm 1,8$ años) y 3 sujetos con disfagia (edad $=68,7 \pm 20,2$ años). En su investigación utilizaron MAR para el análisis de la presión máxima, la tasa de aumento de presión y la duración de la presión por encima del valor inicial para la velofaringe y la base de la lengua, durante la deglución de $5 \mathrm{ml}$ de agua. Un hallazgo interesante presente en los controles sanos y ausente en los participantes con disfagia es un patrón lineal dentro de la deglución, que conecta los picos de presión creados por la velofaringe, la base de la lengua y el EES ${ }^{28}$. El mismo año, otro grupo registró presiones faríngeas con MAR, en 6 sujetos laringectomizados (LT) con disfagia (edad $=61,2 \pm 13,3$ años) y 6 sujetos controles sanos (edad $=1,8 \pm 3,3$ años). Compararon las variables de tiempo y presión para la velofaringe, la mesofaringe y el EES. Todos los participantes deglutieron $10 \mathrm{cc}$ de agua. Los sujetos LT fueron evaluados además con bolos líquidos de 5 y $20 \mathrm{cc}$. A nivel velofaríngeo, los resultados evidenciaron que los sujetos TL comparados con los controles, exhibieron una mayor duración de la presión velofaríngea $(p=0,012)$ y una tasa de aumento de la presión velofaríngea más lenta $(\mathrm{p}=0,005)$; mientras que no hubo diferencias en la presión velofaríngea máxima $(\mathrm{p}=0,821)$ y la presión total generada en la velofaringe $(p=0,163)$. La presión velofaríngea y la duración total de la deglución reflejan la separación de la faringe en conductos distintos para el aire y los alimentos, lo que garantiza el paso exitoso del bolo sin la necesidad de respiración ${ }^{29}$.

La heterogeneidad (edad, sexo, raza) de las poblaciones evaluadas en las investigaciones dificultó el desarrollo de un diseño metodológico conciso que permitiese realizar un análisis comparativo de los estudios. Además, las diferentes metodologías imposibilitaron un metaanálisis entre los estudios.

Con relación a los instrumentos utilizados para la evaluación de la fisiología del esfínter velofaríngeo durante la deglución, algunos estudios utilizan métodos más precisos como la electromiografía intramuscular ${ }^{14-16,20,23}$ y la manometría de alta resolución ${ }^{17-19,21-24}$. Si bien ambos procedimientos entregan información sobre el funcionamiento del EVF, es importante señalar que como se mostró en los resultados, ambas evaluaciones pueden tener una relación directa o inversa dependiendo de la tarea evaluada. Los otros estudios no tan recientes, utilizaron métodos precisos, pero no tan específicos para la evaluación funcional del 
mecanismo velofaríngeo, como el Radio $\mathrm{X}^{12} \mathrm{y}$ la videofluoroscopía ${ }^{13}$.

En cuanto a las tareas utilizadas para evaluar la función del EVF durante la deglución, todos los estudios solicitan verbalmente la deglución de diferentes elementos, entre ellos, saliva o en $\operatorname{seco}^{12,13}$, agua ${ }^{12,15,16,19,23}$, mezcla de bario y agua ${ }^{13}$, té verde ${ }^{20}$, a diferentes volúmenes $^{13,15,16,20}$, viscosidades ${ }^{20}$, consistencias ${ }^{25}$, temperaturas frías ${ }^{17,21,22}$ y temperaturas calientes ${ }^{21}$. Además, se utilizan maniobras ${ }^{19}$, posturas ${ }^{22,24}$ y entrenamientos respiratorios ${ }^{23}$. Ningún artículo justifica de forma experimental o clínica la selección del bolo. Además, no todos los experimentos especifican el utensilio con el cual se entregó el bolo a los participantes. Por último, el efecto de las maniobras, posturas, entrenamientos, cantidades, viscosidades y temperaturas de los bolos, entregan datos importantes que contribuyen a la comprensión de la fisiología y fisiopatología del EVF en la deglución orofaríngea.

\section{Conclusión}

Durante la deglución, el paladar blando se eleva para entrar en contacto con la pared posterior de la faringe, cerrando la nasofaringe. Debido al desconocimiento respecto de cómo ocurre este fenómeno biomecánico, el propósito de esta investigación fue describir la fisiología del esfínter velofaríngeo durante la deglución, mediante una revisión sistemática de literatura.

Los hallazgos de esta investigación evidenciaron que el esfínter velofaríngeo cumple un rol importante en la fase faríngea de la deglución, específicamente separando las cavidades nasal y oral, mediante la activación y relajación sincrónica de distintos músculos, lo cual permite generar cambios en las presiones faríngeas, necesarios para transportar la saliva o el bolo alimenticio desde la boca al esófago.

La mayoría de los estudios analizados en esta revisión son difícilmente replicables debido a lo inespecífico de los procedimientos descritos y los diversos equipamientos utilizados, como lo es el caso de la EMG intramuscular, a diferencia tal vez de la manometría de alta resolución, que debido a su disponibilidad clínica puede ser una herramienta útil que permita dar continuidad a las investigaciones en el área.

Se concluye que la evidencia disponible sobre la fisiología del EVF en la deglución aún es escasa, debido al bajo número de participantes en los estudios y la heterogeneidad de los materiales y métodos. Por esto, es necesario continuar el desarrollo de estudios que describan la fisiología del EVF durante la deglución, con métodos reproducibles como la manometría de alta resolución, la videofluoroscopía y la resonancia magnética tridimensional. La integración del conocimiento permitirá describir de mejor forma la actividad del esfínter velofaríngeo en la deglución, la succión, la masticación, la respiración y el habla.

\section{Bibliografía}

1. Perry J. Anatomy and physiology of the velopharyngeal mechanism. Semin Speech Lang. 2011;32:83-92. doi: 10.1055/s-0031-1277712.

2. Palomares M, Inostroza F, Villarroel C, Urzúa M. Intervención Quirúrgica y Protésica de la IVF en la Fisura Labiopalatina. En: Moya M, Susanibar F, Valdés C, eds, Evaluación e Intervención Logopédica en Motricidad Orofacial y áreas afines. Madrid: Editorial EOS, 2019;187-200.

3. Raol N, Hartnick C. Anatomy and physiology of velopharyngeal closure and insufficiency. Adv Otorhinolaryngol. 2015;76:1-6. doi: $10.1159 / 000368003$.

4. Vuono I, Zanoteli E, de Oliveira A, y cols. Histological analysis of palatopharyngeal muscle from children with snoring and obstructive sleep apnea syndrome. Int J Pediatr Otorhinolaryngol. 2007;71:283-290. doi: 10.1016/j.ijporl.2006.10.019.

5. De Bellis M, Pagni F, Ronchi S, y cols. Immunohistochemical and histomorphometric study of human uvula innervation: a comparative analysis of non-snorers versus apneic snorers. Sleep Breath. 2012;16:1033-1040. doi: 10.1007/s11325-011-0597-7.

6. Croft C, Shprintzen R, Rakoff S. Patterns of velopharyngeal valving in normal and cleft palate subjects: a multi-view videofluoroscopic and nasendoscopic study. Laryngoscope. 1981;91:265-271. doi: 10.1288/00005537-198102000-00015.

7. Liu H, Warren D, Dalston R. Increased nasal resistance induced by the pressure-flow technique and its effect on pressure and airflow during speech. Cleft Palate Craniofac J. 1991;28:261-266. doi: 10.1597/1545-1569_1991_028_0261_inribt_2.3.co_2.

8. Nohara K, Tachimura T, Wada T. Levator veli palatini muscle fatigue during phonation in speakers with cleft palate with borderline velopharyngeal 
incompetence. Cleft Palate Craniofac J. 2006;43:103107. doi: 10.1597/04-152.

9. Perry J, Sutton B, Kuehn D, Gamage J. Using MRI for assessing velopharyngeal structures and function. Cleft Palate Craniofac J. 2014;51:476-485. doi: $10.1597 / 12-083$.

10. Matsuo K, Hiiemae K, Palmer J. Cyclic motion of the soft palate in feeding. J Dent Res. 2005;84:39-42. doi: $10.1177 / 154405910508400106$.

11. Urrútia G, Bonfill X. Declaración PRISMA: una propuesta para mejorar la publicación de revisiones sistemáticas y metaanálisis [PRISMA declaration: a proposal to improve the publication of systematic reviews and meta-analyses]. Med Clin (Barc). 2010;135:507-511. doi: 10.1016/j.medcli.2010.01.015.

12. Hamlet S, Momiyama Y. Velar activity and timing of eustachian tube function in swallowing. Dysphagia. 1992;7:226-233. doi: 10.1007/bf02493474.

13. Kahrilas P, Lin S, Chen J, Logemann J. Oropharyngeal accommodation to swallow volume. Gastroenterology. 1996;111:297-306. doi: 10.1053/gast.1996.v111. pm8690194.

14. Picciotti P, Della Marca G, Restuccia D, Rigante M, Di Nardo W, Scarano E. Tensor veli palatini electromyography with surface electrode applied transnasally. Acta Otorhinolaryngol Ital. 2005;25:120124.

15. Tachimura T, Ojima M, Nohara K, Wada T. Change in palatoglossus muscle activity in relation to swallowing volume during the transition from the oral phase to the pharyngeal phase. Dysphagia. 2005;20:32-39. doi: 10.1007/s00455-004-0022-5.

16. Tachimura T, Okuno K, Ojima M, Nohara K. Change in levator veli palatini muscle activity in relation to swallowing volume during the transition from the oral phase to pharyngeal phase. Dysphagia. 2006;21:713. doi: 10.1007/s00455-005-9005-4.

17. Takasaki K, Umeki H, Enatsu K, y cols. Investigation of pharyngeal swallowing function using highresolution manometry. Laryngoscope. 2008;118:17291732. doi: 10.1097/MLG.0b013e31817dfd02.

18. Hoffman M, Ciucci M, Mielens J, Jiang J, McCulloch T. Pharyngeal swallow adaptations to bolus volume measured with high-resolution manometry. Laryngoscope. 2010;120:2367-2373. doi: 10.1002/ lary.21150.

19. Hoffman M, Mielens J, Ciucci M, Jones C, Jiang J, McCulloch T. High-resolution manometry of pharyngeal swallow pressure events associated with effortful swallow and the Mendelsohn maneuver. Dysphagia. 2012;27:418-426. doi: 10.1007/s00455-
011-9385-6.

20. Okuno K, Tachimura T, Sakai T. Influences of swallowing volume and viscosity on regulation of levator veli palatini muscle activity during swallowing. J Oral Rehabil. 2013;40:657-663. doi: 10.1111/joor.12071.

21. Matsubara K, Kumai Y, Samejima Y, Yumoto E. Swallowing pressure and pressure profiles in young healthy adults. Laryngoscope. 2014;124:711-717. doi: 10.1002/lary.24311.

22. Matsubara K, Kumai Y, Kamenosono Y, Samejima Y, Yumoto E. Effect of three different chin-down maneuvers on swallowing pressure in healthy young adults. Laryngoscope. 2016;126:437-441. doi: 10.1002/ lary. 25552 .

23. Hutcheson K, Hammer M, Rosen S, Jones C, McCulloch T. Expiratory muscle strength training evaluated with simultaneous high-resolution manometry and electromyography. Laryngoscope. 2017;127:797-804. doi: 10.1002/lary.26397.

24. Rosen S, Abdelhalim S, Jones C, McCulloch T. Effect of Body Position on Pharyngeal Swallowing Pressures Using High-Resolution Manometry. Dysphagia. 2018;33:389-398. doi: 10.1007/s00455-017-9866-3.

25. Pongpipatpaiboon K, Inamoto Y, Saitoh E, Kagaya H, Shibata S, Aoyagi Y, Fujii N, Palmer J, Fernández M. Pharyngeal swallowing in older adults: Kinematic analysis using three-dimensional dynamic computed tomography. J Oral Rehabil. 2018;45:959-966. doi: 10.1111/joor.12703.

26. Palmer J, Kuhlemeier K, Tippett D, Lynch C. A protocol for the videofluorographic swallowing study. Dysphagia. 1993;8:209-14. doi: 10.1007/BF01354540.

27. Giannantoni N, Minisci M, Brunetti V, Scarano E, Testani E, Vollono C, De Corso E, Bastanza G, D'Alatri L, Della Marca G. Evaluation of pharyngeal muscle activity through nasopharyngeal surface electromyography in a cohort of dysphagic patients with acute ischaemic stroke. Acta Otorhinolaryngol Ital. 2016;36:295-299. doi: 10.14639/0392-100X-1124.

28. Mielens J, Hoffman M, Ciucci M, Jiang J, McCulloch T. Automated analysis of pharyngeal pressure data obtained with high-resolution manometry. Dysphagia. 2011;26:3-12. doi: 10.1007/s00455-0109320-2.

29. Lippert D, Hoffman M, Britt C, Jones C, Hernandez J, Ciucci M, McCulloch T. Preliminary Evaluation of Functional Swallow After Total Laryngectomy Using High-Resolution Manometry. Ann Otol Rhinol Laryngol. 2016;125:541-9. doi: $10.1177 / 0003489416629978$. 